\title{
NOVEL PARAMETRIC-EFFECT MEMS AMPLIFIERS/TRANSDUCERS
}

\author{
Jean-Pierre Raskin, Andrew R. Brown and Gabriel M. Rebeiz \\ The University of Michigan, Radiation Laboratory, Ann Arbor, MI 48109-2122, USA \\ Butrus T. Khuri-Yakub \\ Stanford University, Edward L. Gizton Laboratory, CA 94305, USA.
}

\begin{abstract}
A parametric effect amplifier has been built at $200 \mathrm{kHz}$ (input) and $1.84 \mathrm{MHz}$ (output) using a MEMS time-varying capacitor. The capacitor is composed of a thin low stress metallized silicon-nitride diaphragm and is pumped by a large signal voltage at $1.64 \mathrm{MHz}$. This results in a large change in the capacitance, and parametric amplification of an input signal at $200 \mathrm{kHz}$. To our knowledge, this device is the first-ever mechanical up-converter parametric-effect amplifier, with an up-conversion ratio of 9:1. Its main advantages are the absence of any resistive and $I / f$ noise, and most importantly, it provides gain at the transducer level. Some advantages over CMOS based electronic amplifiers are the possibility to operate at very high temperatures $\left(200-600^{\circ} \mathrm{C}\right)$, under high particle bombardment (nuclear applications), and very low noise operation due to the absence of thermal and shot noise in parametric amplifiers.
\end{abstract}

\section{INTRODUCTION}

Parametric amplifiers, based on non-linear reactances such as capacitors and inductors, have been extensively used in the 1960's for amplification, frequency up- and down-conversion and oscillators at microwave frequencies [1], [2]. Briefly, parametric devices transfer the power from the pump frequency $\left(\omega_{p}\right)$ to the signal frequency $\left(\omega_{s}\right)$ or to the up-conversion frequency $\left(\omega_{u}\right)$, thereby resulting in gain. Parametric amplifiers were commonly built using varactor diodes for microwave applications. Since parametric amplifiers are not resistive based, they do not suffer from the Johnson and $1 / f$ noise. Simple parametric amplifiers, using the negative resistance effect due to the non-linear reactance, resulted in narrowband amplifiers with a bandwidth of 1-6\%, depending on its gain. On the other side, parametric amplifiers using the parametric up-conversion effect, resulted in amplifiers with a bandwidth of 20-40\%. However, parametric up-conversion as not used since the frequency needed to be downconverted again using a microwave mixer, thereby introducing loss and noise in the system. With the introduction of MESFETs presenting excellent gain and noise characteristics at microwave frequencies, parametric amplifiers were abandoned and to our knowledge, they are not use anymore in any microwave applications. Frequency conversion (doublers, triplers) using a time-varying capacitance (varactor diodes) is still used today, but this effect results in a typical conversion loss of $-4 \mathrm{~dB}$ to $-10 \mathrm{~dB}$ (or a minimum conversion loss of $0 \mathrm{~dB}$ from the Manley-Rower equations) and is not called parametric amplification. This last decade, the parametric amplification has been used mostly in optical systems [3], [4]. In this paper, we present the interest of a novel MEMS parametric-effect for providing gain at the transducer level.

\section{PARAMETRIC AMPLIFICATION THEORY}

In 1956, Manley and Rowe derived a pair of equations governing the power balance of passive and lossless one-port devices with an arbitrary nonlinear characteristics [5]. As an example of such a device we may think of a deal varactor diode, of a Josephson junction or of a variable plate capacitor. If such a device forms a part of a parametric circuit is simultaneously fed by a signal source at frequency $f_{s}$ and a pump source at frequency $f_{p}$. Due to its nonlinear characteristics, time varying currents and voltages are generated, in principle, at all combination frequencies: $f_{n, m}=n f_{s}+$ $m f_{p}$ with $n, m=-\infty \ldots \infty$. Manley and Rowe have shown that the following pair of equations holds:

$$
\times{ }_{n=0 m=-\times} \frac{n P_{n, m}}{n f_{s}+m f_{p}}=0 \quad \text { (1) and }{ }_{m=0 n=-\times}^{\times} \frac{m P_{n, m}}{n f_{s}+m f_{p}}=0
$$

A general understanding of parametric circuits would be very difficult if all these frequencies were unconditionally necessary for the different modes of operation. This is not the case, however, and on the contrary, really not desirable. Actually, in practice, only a single combination frequency $\left(f_{2}\right)$ is kept besides the signal frequency $f_{s}$ and the pump frequency $f_{p}$, the other combination frequencies are filtered. The two most simple choices for $f_{2}$ are: frequency-noninverting case: $f_{2}=f_{u}=f_{p}+f_{s}$ and frequencyinverting case: $f_{2}=f_{l}=f_{p}-f_{s}$. In these cases, from the general results (1)-(2), the available power gain for both cases can be expressed respectively by:

$$
G_{u}=\frac{P_{u}}{P_{s}}=-\frac{f_{p}+f_{s}}{f_{s}} \quad \text { (3) and } G_{l}=\frac{P_{l}}{P_{s}}=-\frac{f_{p}-f_{s}}{f_{s}}
$$

Relations (3)-(4) show that a parametric amplifier fed by an input signal $f_{s}$ and pumped at a frequency $f_{p}$ can present a large gain if $f_{p}$ $\gg f_{s}$.

Considering the time-varying capacitance as a linear four-pole Rowe [6] has calculated the gain, the matching conditions, the bandwidth and the sensitivity for the inverting and noninverting cases. Because the inverting down- and up-converters are potentially unstable and the inverting negative resistance amplifier (degenerate case: $f_{p}=2 f_{s}$ ) requires an isolator (circulator) for operation since the source and the load are at the same port, the noninverting up-converter has been preferred as demonstrator for our study. In fact, as described by Rowe, the noninverting upconverter (signal frequencies $f_{s}$ and $f_{u}=f_{p}+f_{s}$ ) is stable device and yields maximum gain with matched source and load. Under matched conditions the power gain is equal to the ratio of output to input frequencies (3); for widely separated signal frequencies the up-converter has substantial gain. Only a relatively small amount of nonlinearity is required to attain a bandwidth equal to the lowsignal frequency. Since the source and load are matched the sensitivity is zero. Fig. 1 represents the scheme of the parametric up-converter which will be analyzed and built using the latest MEMS technologies.

Since we consider ideal input and output filters, all other sidebands are terminated with an open-circuit. In Fig. $1 C(t)$ is the timevarying capacitance, $R_{c}$ is the parasitic series resistance of the time-varying capacitor, $R_{G}$ and $R_{L}$ the source and the load resistances, respectively, and, $R_{s}+j \omega L_{s}$ and $R_{u}+j \omega L_{u}$ are, respectively, the terminal impedances added for matching purposes. For conceptual purposes, the circuit model of Fig. 1 can be analyzed as a linear four-terminal scheme. The transducer gain is defined as the ratio of the actual power output $\left(\left|I_{L}\right|^{2} . R_{L}\right)$ to the 
available power input $\left(\left|V_{G}\right|^{2} / 4 R_{G}\right)$ and is calculated by the standard linear circuit techniques.

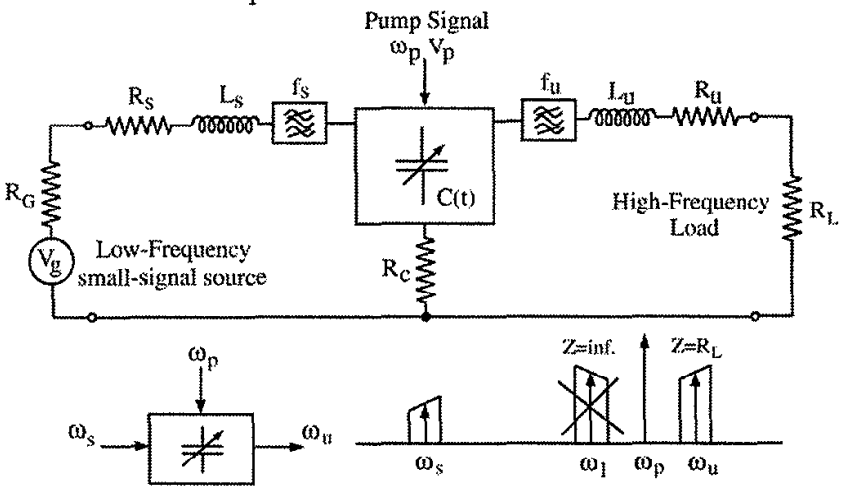

Figure 1. Equivalent model and amplitude spectra for a generic parametric up-converter.

Blackwell and Kotzebue in [7] have solved the transducer gain corresponding to equivalent circuit presented in Fig. 1.

$$
G_{t}=\frac{4 R_{G} R_{L}\left|I_{L}\right|^{2}}{\left|V_{G}\right|^{2}}=\frac{4 R_{G} R_{L}\left|Z_{21}\right|^{2}}{\left|\left(Z_{11}+Z_{T 1}\right)\left(Z_{22}+Z_{T 2}\right)-Z_{12} Z_{21}\right|^{2}}
$$

with $Z_{i j}$ the two-port $Z$ parameters of the time-varying capacitor $C(t), Z_{I I}=$ total external circuit impedance at $f_{s}: Z_{T l}=X_{s}+R_{s}+R_{G}$ $+R_{c}$ and $Z_{T 2}=$ total external circuit impedance at $f_{u}: Z_{T 2}=X_{u}+R_{u}$ $+R_{L}+R_{c}$. Neglecting the losses of the matching inductances $L_{s}$ and $L_{u}\left(R_{s}=R_{u}=0\right)$, the maximum transducer gain and bandwidth are reached under the matched conditions defined as follows:

$$
\begin{aligned}
R_{G} & =R_{L}=R_{c} \sqrt{1+\frac{\gamma^{2}}{\omega_{s} \omega_{u} C^{2} R_{c}{ }^{2}}} \\
X_{s} & =\frac{1}{\omega_{s} C} \\
X_{u} & =\frac{1}{\omega_{u} C} \\
\text { with } \gamma & =\gamma_{1}\left(+\gamma_{1}^{2}\right)\left(1-\gamma_{2}\right) \text { and } C=C_{0}\left(1-2 \gamma_{1}^{2}\right)
\end{aligned}
$$

And it is given by:

$$
G_{t}=\frac{f_{p}+f_{s}}{f_{s}} \frac{x}{[+\sqrt{1+x}]}
$$

where $x=\left(f_{p}+f_{s}\right) / f_{s}(\gamma Q)^{2}$ with $Q$ the effective quality factor of the time-varying capacitor, $Q=I /\left(\omega_{s} C R_{c}\right)$. It is interesting to note that if we consider a lossless time-varying capacitor $(Q \rightarrow \infty$, i.e. $R_{c}=0$ ), the transducer gain tends to the ratio $\left(f_{p}+f_{s}\right) / f_{s}$ which is in accordance with the power gain defined from the Manley and Rowe relationships (3) for the noninverting case.

Under the matching conditions defined above, the maximum bandwidth is given by:

$$
B W=\gamma \sqrt{2 f_{u} f_{s}}
$$

\section{MEMS PARAMETRIC UP-CONVERTER}

The parametric effect results from a time-varying capacitor which consists of a metallized membrane (top electrode) suspended above a heavily doped silicon bulk (bottom electrode). By pumping the movable top electrode with a large signal voltage at the pump frequency $\left(\omega_{p}\right)$, one generates a time-varying capacitance: $C(t)=C_{0}\left(1+2 \gamma_{1} \cos \left(\omega_{p} t+\varphi_{1}\right)+2 \gamma_{2} \cos \left(2 \omega_{p} t+\varphi_{2}\right)+\ldots\right)(11)$
The fabrication process is summarized in Fig. 2 and more details can be found in [8].

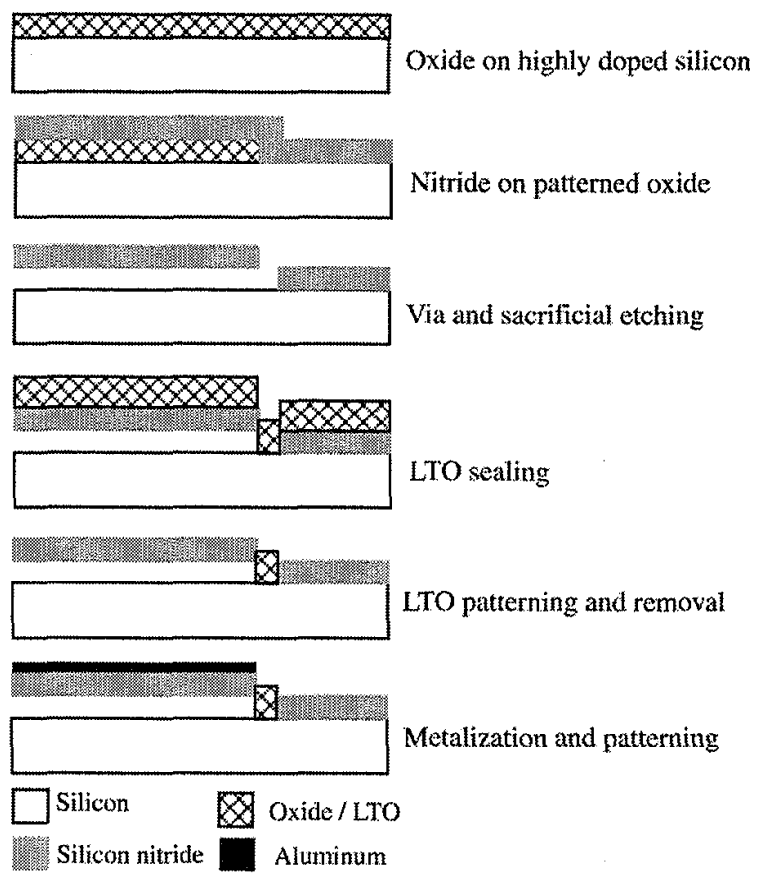

Figure 2. Fabrication steps of the MEMS capacitor array.

\section{A. Static Analysis}

The external load in that case is an electrostatic force $F_{e}$ induced by the source voltage $V_{d c}$ applied between the two electrodes of the MEMS capacitor. Fig. 3 shows the capacitance variation of the MEMS capacitor versus dc bias conditions. The suspended nitride membrane is $0.6 \mu \mathrm{m}$ thick, $50 \mu \mathrm{m}$ radius and with a residual stress of $170 \mathrm{MPa}$. The total device area is $1 \mathrm{~cm}^{2}$. The calculated collapse potential for the measured structure is $85 \mathrm{~V}$. It is important to work under that limit de potential to avoid the collapse of the membrane.

\section{B. Small-Signal Analysis}

In the linear case, we assume that the microstructure displacements $(x)$ are small compared to the physical gap dimensions of the structure $\left(g_{0}\right)$. With other words, if the applied external force has a sine wave form, the movement of the mass will also be sinusoidal at the same frequency (linear system). The linear analysis is useful for determining the mechanical resonant frequency and quality factor of the movable structure versus frequency.

Under small-signal conditions, the capacitor is driven by a small ac voltage $v_{a c}$ superimposed on the bias $\left(V_{d c}\right)$ which induces a small ac displacement $x$. The dynamics of the resonator under small signal conditions are approximately determined by the second order ordinary differential equation:

$m \ddot{x}+b \dot{x}+k x=f_{e}$

where $m$ is the mass of the suspended diaphragm, $b$ the damping coefficient, $k$ the spring constant of the system and $f_{e}$ the small signal ac electrostatic force expressed by:

$$
f_{e}=\frac{f F_{e}}{f V_{d c}} v_{a c}=\frac{C_{0} V_{d c} v_{a c}}{g_{0}}
$$

Since the resulting equation is linear, we can assume that all variables can be represented as phasors yielding:

$$
\hat{x}=\frac{-C_{0} V_{d c}}{g_{0} m \omega_{0}^{2}} \sqrt{\frac{\hat{v}_{a c}}{1-\omega^{2} / \omega_{0}^{2}+j \omega b / m \omega_{0}^{2}}}
$$


where $\omega_{0}=\sqrt{k / m}$ is the resonant frequency.

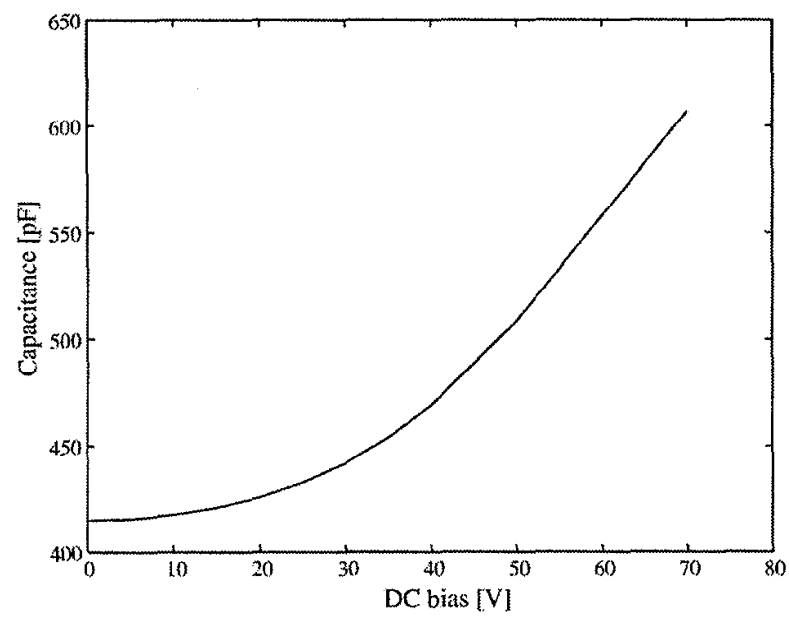

Figure 3. Measured capacitance variation versus dc voltage.

The device resonance can be detected by sensing the device impedance. In fact, by analogy the spring-mass-damper system can be represented by the equivalent small-signal electrical circuit in Fig 4. In this scheme $R_{c}$ represents the conductor losses in the capacitor plate. The capacitor current is expressed as $\hat{i}_{a c}=\left(Y_{0}+Y_{x}\right) \hat{N}_{a c}$ where

$$
\begin{aligned}
& Y_{0}=j \omega C_{0} \\
& Y_{x}=\frac{j \omega C_{x}}{1-\omega^{2} / \omega_{0}^{2}+j \omega C_{x} R_{x}}
\end{aligned}
$$

From the measurement of its electrical transfer function, the mechanical properties of the resonant MEMS capacitor such as the quality factor $Q$ and the resonant frequency are extracted. Under a dc bias of $60 \mathrm{~V}$, the capacitor membrane has a resonant frequency of $1.64 \mathrm{MHz}$ and a $Q$ of 18 . From the plot of the real (Fig. 5) and imaginary (Fig. 6) parts of the parameter $Y_{12}$, the series parasitic resistance $R_{c}$, the parallel capacitance $C_{0}$ and the equivalent electrical lumped elements of the resonator $L_{x} C_{x}$ and $R_{x}$ are extracted (see Figs. 5 and 6).

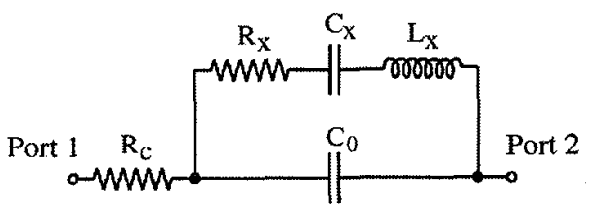

Figure 4. Small-signal electrical model of the MEMS capacitor.

\section{Large Signal Analysis}

If a large pump signal voltage is applied, the displacements of the suspended diaphragm become non-negligible compared to the default gap $\left(g_{0}\right)$, then non-linear behaviors appear and a timevarying capacitance $C(t)$ is generated. In order to minimize the magnitude of the pump signal voltage and then the power consumption, the pump frequency $f_{p}$ is chosen equal to the nature resonant frequency of the capacitor plate. More the displacements are large more the values of the Fourier series coefficients $\gamma_{n}$ are important. Their extraction is an essential step for designing a parametric amplifier/up-converter. Two extraction methods have been developed depending on the importance of the parasitic conductor series resistance $R_{c}$ of the MEMS capacitor plate. In fact, if $R_{c}$ is small such as $j \omega R_{c} C_{o} \ll 1$, coefficients $\varphi_{n}$ tend to zero and $\gamma_{n}$ can be extracted directly from the current spectrum

magnitude given by a spectrum analyzer. If $j \omega R_{c} C(\omega) \ll 1$, with other words, if $R_{c}$ can be neglected compared to $(j \omega C(\omega))^{-2}$, the current through the time-varying MEMS capacitor, when a voltage signal $v(t)=V_{d c}+v_{a c} \cos \left(\omega_{p} t\right)$ is applied, can be expressed in the frequency domain by:

$$
I(\omega)=j \omega(C(\omega) * V(\omega))
$$

Solving the convolution product, we obtain the following expressions for the three first harmonics:

$$
\begin{aligned}
& I\left(\omega_{p}\right)=v_{a c} j \omega_{p} \pi C_{0}\left(1+\gamma_{2}\right)+V_{d c} j \omega_{p} 2 \pi \gamma_{1} C_{0} \\
& I\left(2 \omega_{p}\right)=v_{a c} j 2 \omega_{p} \pi C_{0}\left(\gamma_{1}+\gamma_{3}\right)+V_{d c} j 2 \omega_{p} 2 \pi \gamma_{2} C_{0} \\
& I\left(3 \omega_{p}\right)=v_{a c} j 3 \omega_{p} \pi C_{0} \gamma_{2}+V_{d c} j 3 \omega_{p} 2 \pi \gamma_{3} C_{0}
\end{aligned}
$$

In that case, from the current magnitude measured with a spectrum analyzer and the analytical relations (18)-(20), the Fourier series coefficients $\gamma_{n}$ are extracted. On the other hand, if $R_{c}$ is not negligible the current spectrum is not sufficient to extract the coefficients $\gamma_{n}$ and $\varphi_{n}$ of $C(t)(11)$. Actually, in that case we need a measurement of the current phase through the MEMS capacitor. This current phase information can be provided by the measurement of the voltage $v(t)$ across $C(t)$ and the current $i(t)$ flowing through it with a digital scope as explained below.

The current $i(t)$ is obtained by measuring the voltage across a external resistor $R_{c x t}$ connected in series with the time-varying capacitor. Knowing the value of the parasitic resistor $R_{c}$ (extracted from the small-signal analysis) the voltage across $C(t)$ is given by:

$$
v(t)=v_{t}(t)-i(t)\left(R_{\text {ext }}+R_{c}\right)
$$

Then the time-varying capacitance can be expressed as:

$$
C(t)=\frac{i(t) d t+C_{0} V_{d c}}{v(t)}
$$

Finally, considering the extracted numerical function $C(t)$ as a periodic time function, it can be decomposed into Fourier series for determining the coefficients $\gamma_{n}$ and $\varphi_{n}$. Fig. 7 shows the measured harmonics level at the $50 \Omega$ input of the spectrum analyzer for the MEMS time-varying capacitance pumped with an ac large signal $v(t)=60+15 \cos \left(\omega_{p} t\right)$ with $\omega_{p}=1.64 \mathrm{MHz}$. Unfortunately, due to the pretty high value of $R_{c}=50 \Omega$ compared to the equivalent impedance $((j \omega)(\omega))^{-1}=400 \Omega$ ) of the MEMS capacitor, it is not possible to characterize accurately its non-linear behavior from the only measurement of its current spectrum. In that casc, we have to use the second extraction method based on the measurements of voltages versus time with a scope. From the measured characteristic $C(t)$, the coefficients $\gamma_{n}$ and $\varphi_{n}$ extracted are: $\gamma_{3}=$ $0.22, \gamma_{2}=0.05, \gamma_{3}=0.01, \varphi_{1}=7^{\circ}, \varphi_{2}=3^{\circ}$ and $\varphi_{3}=0.85^{\circ}$.

From the measured characteristics $\left(\omega_{0}, Q, \gamma_{n}, \varphi_{n}\right.$, etc.) of the timevarying capacitor, the input and output matching networks and filters are designed to maximize the gain of the built MEMS parametric up-converter. Fig. 8 shows the measured and simulated transducer gain of the parametric up-converter versus the load resistance. A good agreement is obtained between measurements and simulations. It is interesting to note that the optimum transducer gain achieves effectively the available power gain $G_{u}=1.84 \mathrm{MHz} / 200 \mathrm{kHz}=9.6 \mathrm{~dB}$ when $R_{c}$ tends to zero. The measured optimum load of $140 \Omega$ is in accordance with the value $(133 \Omega$ ) calculated with the analytical relationship (6). Fig. 9 presents the measured transducer gain versus the coefficient $\gamma_{3}$ which depends on the pump signal magnitude. For these measurements, the matching networks and input/output filters has been optimized for $\gamma_{I}=0.22$. As predicted by the simulations, the transducer gain of the MEMS parametric up-converter increases with $\gamma_{I}$.

\section{CONCLUSION}


Parametric amplificrs can be built for sensors (thermal, acceleration, chemical, etc.) without any CMOS electronics. This means that a pre-amplification stage ca be provided for non CMOS sensors before sending the signal to a CMOS wafer for more gain and processing.

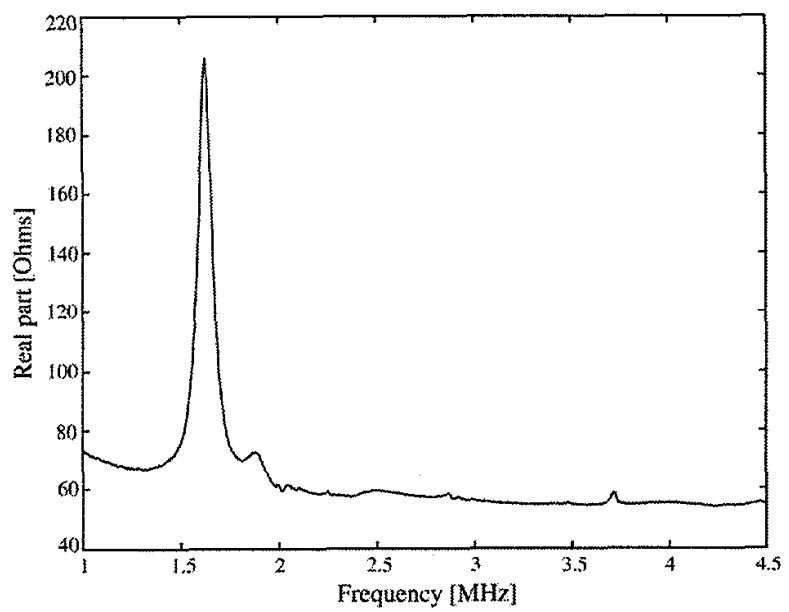

Figure 5. Measured real part of $1 / Y_{12}$ versus frequency. Extracted value of $R_{c}$ is $52 \Omega$.

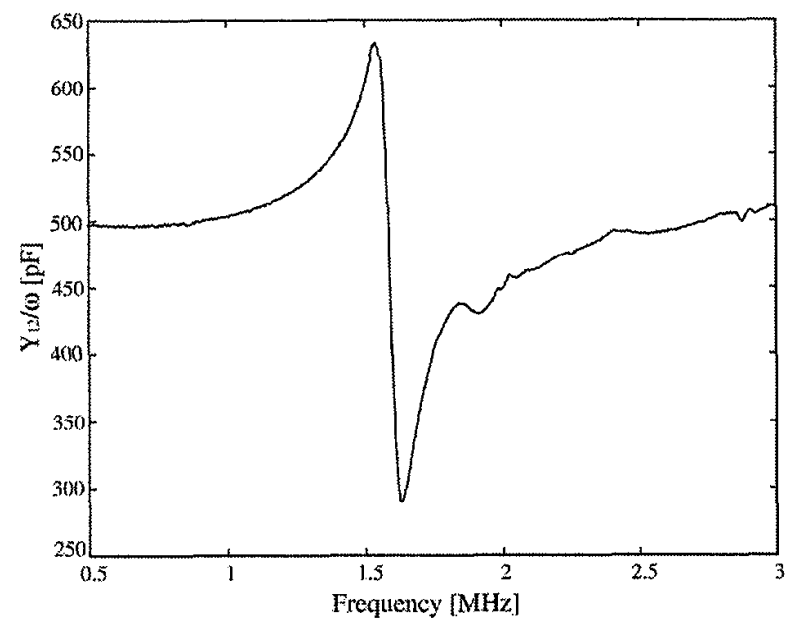

Figure 6. Measured equivalent capacitance $\left(Y_{12} / \omega\right)$ around the resonant frequency. Extracted values of $C_{0}, C_{x}, L_{x}$ and $R_{x}$ are, respectively, $500 \mathrm{pF}, 32.5 \mathrm{pF}, 0.29 \mathrm{mH}$ and $166 \Omega$.

\section{REFERENCES}

[1] George L. Matthaei, IRE Trans. on MTT, pp. 23-28, Jan. 1961.

[2] E. Sard et al, IEEE Trans. on MTT, pp. 608-618, Dec. 1966.

[3] Y. J. Ding and J. B. Khurgin, IEEE J. of Quantum Electronics, pp. 1574-1582, Sept. 1996.

[4] G. M. Gal et al., IEEE J. of Selected Topics in Quanturn Electronics, pp. 224-229, Feb. 1998.

[5] J. M. Manley and H. E. Rowe, Proceedings of the IRE, pp. 904914, July 1956.

[6] H. E. Rowe, Proceedings of the IRE, pp. 850-860, May 1958.

[7] L. A. Blackwell and K. L. Kotzebue, "Semiconductor-diode parametric amplifiers", Prentice-Hall, Inc., N.J., 1961.

[8] Xuecheng Jin, et al., IEEE Journal of Microelectromechanical Systems, vol. 8, no. 1, pp. 100-114, March 1999.

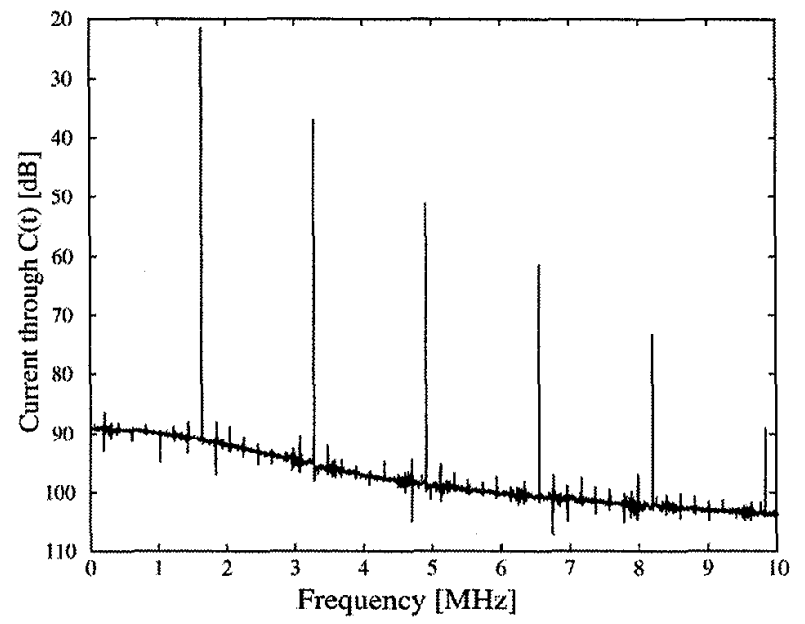

Figure 7. Measured spectrum of the current flowing through the MEMS time-varying capacitor for $V_{d c}=60 \mathrm{~V}$ and $v_{a c}=15 \cos \left(2 \pi f_{p}\right.$ t), with $f_{p}=1.64 \mathrm{MHz}$.

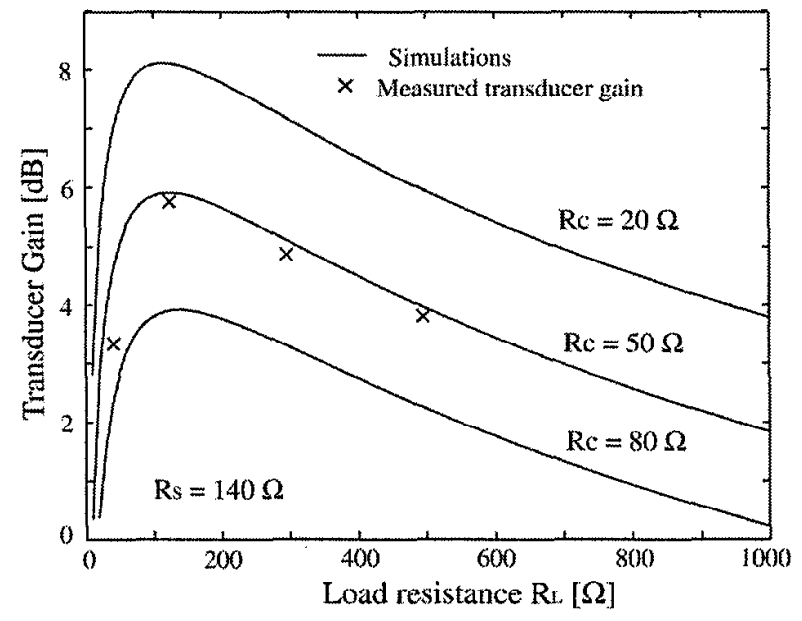

Figure 8. Measured parametric up-converter transducer gain versus the load resistance value $\left(f_{s}=200 \mathrm{kHz}\right.$ and $\left.f_{p}=1.64 \mathrm{MHz}\right)$.

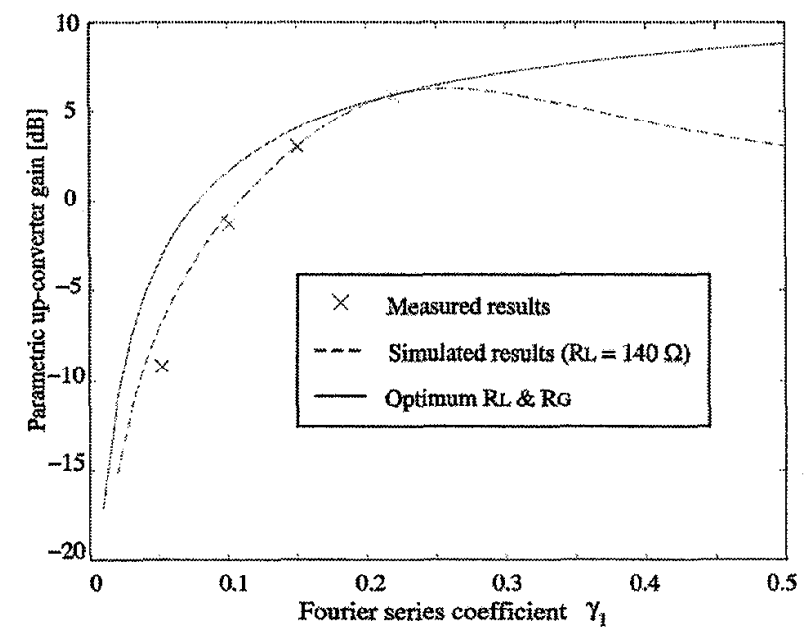

Figure 9. Measured parametric up-converter transducer gain versus $\gamma_{1}\left(f_{s}=200 \mathrm{kHz}\right.$ and $\left.f_{p}=1.64 \mathrm{MHz}\right)$. 